\title{
Revista Colombiana de

\section{Factores de riesgo para mortalidad en falla cardiaca aguda. Análisis de árbol de regresión y clasificación}

\section{Fernando Fortich, Alejandro Ochoa Morón, Benito Balmaceda de La Cruz, Jairo Rentería Roa, Daniel Herrera Orego, Jairo Gándara, Edison Muñoz O., Gilma Hernández y Juan M. Sénior Sánchez*}

Grupo para el Estudio de las Enfermedades Cardiovasculares -GEEC-, Sección Cardiología, Departamento de Medicina Interna, Universidad de Antioquia, Medellín, Colombia

Recibido el 18 de mayo de 2018; aceptado el 18 de marzo de 2019

Disponible en Internet el 14 de noviembre de 2019

\section{PALABRAS CLAVE}

Falla cardiaca aguda; Pronóstico;

Predictores

\begin{abstract}
Resumen
Introducción: la estimación temprana del riesgo en falla cardiaca aguda puede ayudar en la toma de decisiones clínicas.

Objetivo: identificar los factores de riesgo asociados a mortalidad intrahospitalaria en pacientes con falla cardiaca aguda.

Métodos: análisis de una cohorte retrospectiva de pacientes mayores de 18 años ingresados a hospitalización por falla cardiaca aguda en un hospital de tercer nivel, entre los años 2012 y 2016.

Resultados: se incluyeron 247 pacientes, con edad promedio de 62,8 años; predominó el sexo masculino con un $60 \%$. El $84 \%$ de los pacientes tenía falla cardiaca con fracción de expulsión disminuida (mediana de $25 \%$ ). La mortalidad intrahospitalaria fue de $9,3 \%$ y la acumulada a 30 días y 6 meses posterior al egreso hospitalario fue de 10,9 y $14,1 \%$, respectivamente. Los dos predictores asociados a muerte intrahospitalaria fueron el nitrógeno ureico en sangre (BUN) > $37 \mathrm{mg} / \mathrm{dl}$ (OR: 10,8; 95\% IC: 4,10-28,8) y la presión arterial sistólica (PAS) $\leq 125 \mathrm{~mm} \mathrm{Hg}$ (OR: 3,42; 95\% IC:1,15-10,0). El modelo de árbol de regresión y clasificación (CART) identificó como el mejor predictor de mortalidad los niveles elevados de BUN ( $\geq 32,5 \mathrm{mg} / \mathrm{dl}$ ), seguido por la presión sistólica disminuida $(<97 \mathrm{~mm} \mathrm{Hg}$ ) y finalmente por los niveles elevados de creatinina $(\geq 1,75 \mathrm{mg} / \mathrm{dl})$.

Conclusión: el análisis mediante el CART permite clasificar en forma temprana la probabilidad de muerte por un árbol de riesgo que incluye el BUN $\geq 32,5 \mathrm{mg} / \mathrm{dl}$, la presión sistólica $<97 \mathrm{~mm}$ $\mathrm{Hg}$ y los niveles de creatinina $\geq 1,75 \mathrm{mg} / \mathrm{dl}$.

(c) 2019 Sociedad Colombiana de Cardiología y Cirugía Cardiovascular. Publicado por Elsevier España, S.L.U. Este es un artículo Open Access bajo la licencia CC BY-NC-ND (http:// creativecommons.org/licenses/by-nc-nd/4.0/).
\end{abstract}

\footnotetext{
* Autor para correspondencia.

Correo electrónico: mmbt@une.net.co (J.M. Sénior Sánchez).
} 


\section{KEYWORDS}

Acute heart failure;

Prognosis;

Predictors

\section{Risk factors for mortality in acute heart failure. A classification and regression tree analysis}

\begin{abstract}
Introduction: The early estimation of risk in acute heart failure may help in the taking of clinical decisions.

Objective: To identify the risk factors associated with in-hospital mortality in patients with acute heart failure.

Methods: An analysis was performed on a retrospective cohort of patients greater than 18 years admitted to a tertiary hospital due acute heart failure between the years 2010 and 2016.

Results: A total of 247 patients were included, with a mean age of 62.8 years, and of which $60 \%$ were male. The large majority $(84 \%)$ of the patients had heart failure with a reduced ejection fraction (median 25\%). The in-hospital mortality was $9.3 \%$, and the accumulated rate at 30 days and 6 months after hospital discharge was $10.9 \%$ and $14.1 \%$, respectively. The two predictors associated with in-hospital death was a blood urea nitrogen (BUN) $>37 \mathrm{mg} / \mathrm{dL}$ (OR: 10.8; $95 \%$ $\mathrm{Cl}: 4.10-28.8)$ and a systolic blood pressure (SBP) $\leq 125 \mathrm{mmHg}$ (OR: $3.42 ; 95 \% \mathrm{Cl}: 1.15-10.0$ ). The classification and regression tree (CART) model identified elevated levels of as the best predictor of mortality, followed by a decreased systolic pressure $(<97 \mathrm{mmHg})$, and finally due to elevated creatinine levels $(\geq 1,75 \mathrm{mg} / \mathrm{dL})$.

Conclusion: The analysis using the classification and regression tree (CART) model can provide an early classification of the probability of death by a risk tree that includes BUN $\geq 32.5 \mathrm{mg} / \mathrm{dL}$, systolic pressure $<97 \mathrm{~mm} \mathrm{Hg}$, and creatinine levels $\geq 1.75 \mathrm{mg} / \mathrm{dL}$.

(c) 2019 Sociedad Colombiana de Cardiología y Cirugía Cardiovascular. Published by Elsevier España, S.L.U. This is an open access article under the CC BY-NC-ND license (http:// creativecommons.org/licenses/by-nc-nd/4.0/).
\end{abstract}

\section{Introducción}

La falla cardiaca aguda es una pandemia de crecimiento acelerado ${ }^{1}$. Se estima que en los Estados Unidos la padecen alrededor de 6 millones de personas y cada año se reportan más de 1 millón de hospitalizaciones atribuibles a esta como causa primaria, así como varios millones de hospitalizaciones que la incluyen como diagnóstico secundario ${ }^{1-3}$. En Colombia, Sénior et al. ${ }^{4}$, describieron 106 pacientes que acudieron a un centro de tercer nivel con diagnóstico de falla cardiaca aguda, con edad promedio de 62,4 años, de los cuales el $52,8 \%$ correspondía a hombres. Las etiologías más frecuentes de falla cardiaca fueron: hipertensiva $45,2 \%$, idiopática $31,1 \%$, isquémica $14,1 \%$, valvular $8,4 \%$, viral y periparto $0,9 \%$. Por su parte, Chaves et al. ${ }^{5}$ hicieron un análisis de los factores predictores de mortalidad y encontraron que el nitrógeno ureico en la sangre (BUN) mayor o igual a $43 \mathrm{mg} / \mathrm{dl}$ fue el único predictor de mortalidad intrahospitalaria y que este, además de la estancia hospitalaria mayor a cinco días y la porción $\mathrm{N}$ terminal del péptido natriurético tipo B (NT-proBNP) mayor o igual a $4.630 \mathrm{pg} / \mathrm{dl}$ fueron predictores de mortalidad a treinta días.

Así, entonces, la mortalidad intrahospitalaria por falla cardiaca aguda se sitúa entre 7,1 y $12,9 \%^{6}$, a 30 días es de $10,7 \%^{7}$, a 6 meses es de $18,7 \%^{3}$ y a un año es de $30-40 \%{ }^{7}$.

Muchas características clínicas individuales, incluido choque cardiogénico, baja presión sistólica al ingreso, hipercalemia, bajos niveles de colesterol, uso de inotrópicos, disfunción ventricular izquierda, insuficiencia renal o edad mayor de 70 años se conocen como predictores independientes de mortalidad entre pacientes hospitalizados por falla cardiaca aguda ${ }^{6}$. El conocimiento de predictores de mortalidad puede ser usado para generar modelos predictivos que ayuden en la toma de decisiones a los clínicos, en particular para identificar pacientes de bajo y alto riesgo ${ }^{7}$, aspecto fundamental en la toma de decisiones de pacientes con falla cardiaca aguda ${ }^{8-11}$.

Los modelos de predicción pueden emplearse en la consejería de pacientes para iniciar la discusión acerca de los cuidados de fin de vida y también para la evaluación de desenlaces de calidad de vida ${ }^{7}$. Los pacientes de bajo riesgo pueden ser egresados del hospital de forma temprana, mientras que los considerados de alto riesgo pueden beneficiarse de un tratamiento más exhaustivo en unidades de cuidados intensivos o especializado ${ }^{7}$.

Fonarow et al., en el estudio The Acute Decompensated Heart Failure National Registry (ADHERE), identificó al nitrógeno ureico en sangre (BUN), a la creatinina y a la presión arterial sistólica como los mejores predictores de mortalidad intrahospitalaria de acuerdo con un árbol de regresión y clasificación (CART) ${ }^{12}$. Por otra parte, Tomcikova et al. ${ }^{6}$, diseñó un árbol de clasificación del AHEAD-Main Registry, empleando tres variables: choque cardiogénico, presión arterial sistólica al ingreso y creatinina sérica. El área bajo la curva (características operativas del receptor o ROC) $)^{13}$ fue de 0,823 , más alta que las descritas del modelo del registro ADHERE propuesto por Fonarow et al. La validación externa de modelos predictivos es una prueba más robusta, desde el punto de vista estadístico, que la validación interna, puesto que evalúa la transportabilidad en términos temporales, geográficos o de escenario, más que la reproducibilidad ${ }^{14}$. 
El objetivo de este estudio fue identificar los predictores de mortalidad intrahospitalaria en pacientes con falla cardiaca aguda en un hospital de tercer nivel de Colombia y desarrollar un método de clasificación simple y amigable para el clínico.

\section{Métodos}

Se hizo un análisis de una cohorte retrospectiva de pacientes adultos mayores de 18 años con diagnóstico de falla cardiaca aguda, que ingresaron al servicio de emergencias de un hospital de alta complejidad, entre los años 2012 y 2016. La falla cardiaca aguda se definió de acuerdo con las categorías de diagnóstico posible y definitivo de los criterios clínicos de Boston (puntaje $\geq 5)^{15,16}$ y se clasificó el tipo de falla cardiaca de acuerdo con las guías del Colegio Americano de Cardiología (ACC)/ Asociación Americana del Corazón, como falla cardiaca con fracción de expulsión reducida cuando la fracción de expulsión del ventrículo izquierdo ( $\mathrm{FEVI}$ ) es menor o igual a $40 \%$ y preservada cuando la $\mathrm{FEVI}$ es mayor a $40 \%$ asociada a disfunción diastólica del ventrículo izquierdo por ecocardiografía Doppler o cateterismo cardiaco ${ }^{17}$. La fracción de expulsión fue determinada durante la hospitalización o se tomó la última determinación dentro de los seis meses previos al ingreso hospitalario. Se excluyeron los casos de falla cardiaca aguda secundaria a síndrome coronario agudo, pacientes embarazadas, miocardiopatía periparto o falla cardiaca derecha aislada. Las historias clínicas fueron revisadas por personal entrenado. Los datos tomados incluyeron información demográfica y clínica, incluyendo el perfil clínico Stevenson ${ }^{18}$, de laboratorio, ecocardiografía y desenlaces clínicos intrahospitalarios y del seguimiento a treinta días y seis meses. En caso de que no hubiese registro en la historia clínica de los desenlaces a treinta días o seis meses se estableció contacto telefónico para la recolección de dicha información. En caso de que un mismo paciente tuviese múltiples hospitalizaciones se registró solo la hospitalización más reciente. Esta investigación se clasificó como estudio "sin riesgo", según el numeral b del Artículo 11, de la resolución 008430 de 1993 del Ministerio de Salud de la República de Colombia ${ }^{19}$. El estudio fue presentado y aprobado por el comité de ética institucional.

\section{Análisis estadístico}

Las características basales de los pacientes del estudio fueron reportadas como medias y medianas, con sus respectivas medidas de dispersión para las variables continuas y porcentajes para las variables categóricas. Se construyó un modelo de regresión logística para identificar los factores asociados a mortalidad intrahospitalaria ingresando las variables según criterio clínico y estadístico. El desenlace primario fue mortalidad intrahospitalaria. Cinco variables predictoras candidatas fueron consideradas en el modelo; estas fueron: BUN al ingreso, sodio sérico al ingreso, presión arterial sistólica al ingreso, etiología isquémica de la falla cardiaca y existencia de bloqueo completo de rama izquierda del haz de His. Se exploró la capacidad discriminativa del modelo a través del área bajo la curva ROC. Se compararon las medianas para las variables BUN, presión arterial sistólica y sodio por medio de la prueba de Mann-Whitney. Las variables BUN
Tabla 1 Características demográficas y comorbilidades

\begin{tabular}{ll}
\hline Característica & $\mathrm{n}=247$ \\
\hline Edad (años, X $\pm \mathrm{DE}$ ) & $62,8 \pm 14,8$ \\
Sexo masculino (\%) & 60,7 \\
Hipertensión arterial (\%) & 72,1 \\
Diabetes mellitus (\%) & 15,8 \\
Enfermedad renal crónica (\%) & 28,3 \\
- Pacientes en diálisis (\%) & 7,3 \\
Enfermedad coronaria previa (\%) & 27,5 \\
Antecedente de falla cardiaca (\%) & 74,1 \\
- Consumo previo de diurético de asa (\%) & 59,9 \\
Enfermedad pulmonar obstructiva crónica (\%) & 29,6 \\
Enfermedad arterial periférica (\%) & 5,8 \\
\hline
\end{tabular}

y presión arterial sistólica se recodificaron de acuerdo con los puntos de corte establecidos en un estudio previo ${ }^{20}$. Para los análisis estadísticos se usó el software Stata, versión 12.

Se construyó un árbol de regresión y clasificación (CART) con base en las variables predictoras definidas adicionando la creatinina con el programa estadístico $\mathrm{R}^{21}$. El análisis CART es una forma de partición binaria que utiliza nodos, los cuales pueden dividirse en dos ramas que obtienen una forma de clasificación más simple de interpretar; es no paramétrico, por lo que no debe cumplir los supuestos respecto a la distribución de las variables con capacidad pronóstica, puede manejar tanto variables numéricas sesgadas o multimodales, como categóricas, con estructura ordinal o no $\operatorname{ordinal}^{22}$.

\section{Resultados}

Los pacientes admitidos por el servicio de urgencias de un Hospital Universitario de alta complejidad en el periodo de enero a agosto de 2016 y aquellos inscritos en el programa de falla cardiaca con admisiones por emergencias a la institución desde el año 2012 fueron ingresados a la base de datos; se excluyeron 10 pacientes con falla cardiaca derecha, 2 por miocardiopatía periparto y 12 por síndrome coronario agudo, obteniéndose un total de 247 pacientes.

\section{Características demográficas e historia médica}

La edad promedio al momento del ingreso fue de $62,8 \pm 14,8$ años, con predominio del sexo masculino (60,7\%). Las comorbilidades más frecuentes fueron: hipertensión arterial (72\%), enfermedad pulmonar obstructiva crónica $(29,6 \%)$, enfermedad renal crónica (28\%), enfermedad coronaria $(27,5 \%)$, diabetes mellitus $(15,8 \%) y$, en menor proporción, enfermedad arterial periférica (5,8\%). Hasta el $74 \%$ de los pacientes tenía el diagnóstico previo de falla cardiaca y el $60 \%$ recibía tratamiento con diurético de asa tipo furosemida (tabla 1).

\section{Características clínicas}

El perfil clínico más frecuente al momento del ingreso fue el húmedo-caliente (Stevenson $\mathrm{B}$ ), presente en el $85 \%$ de los casos, seguido del húmedo-frío (10\%). El $84 \%$ de los pacien- 
Tabla 2 Caracterización de la falla cardiaca

\begin{tabular}{ll}
\hline Característica & $\mathrm{n}=247$ \\
\hline Clasificación clínica Stevenson (\%): & \\
A & 2,40 \\
B & 85,4 \\
C & 10,1 \\
L & 2 \\
Tipo de falla cardiaca (\%): & \\
Fracción de expulsión reducida $\leq 40 \%$ & 84,2 \\
Fracción de expulsión preservada $>40 \%$ & 15,8 \\
Presencia de bloqueo completo de la rama & 21,9 \\
izquierda del haz de His (\%) & \\
- QRS promedio (ms) & 149 \\
Causa de descompensación (\%): & \\
Progresión de falla cardiaca & 27,1 \\
Falla cardiaca aguda de novo & 25,9 \\
Falta de adherencia & 22,6 \\
Fibrilación atrial & 6,1 \\
Infección & 5,3 \\
Otras arritmias & 2 \\
Drogas/alcohol & 2 \\
Otras causas & 9 \\
Etiología de la falla cardiaca (\%): & \\
Hipertensiva & \\
Isquémica & \\
Valvular & 31,6 \\
Causa no clara & 24,3 \\
Dilatada idiopática & 14,5 \\
Congénita & 6,20 \\
Taquicardiomiopatía & 5,38 \\
Chagásica & 4,62 \\
Infiltrativa & 2,85 \\
Otras causas & 2,85 \\
Puntaje en escala de Boston (X, $\pm D E) ~$ & 2,85 \\
Fracción de expulsión (\%, Me, Q1-Q3) & 4,85 \\
& $9,5 \pm 2,08$ \\
& $25(18-37)$ \\
\hline
\end{tabular}

tes tenía falla cardiaca con fracción de expulsión reducida. El bloqueo completo de la rama izquierda del haz de His estuvo presente en el $22 \%$ de los pacientes. Dentro los factores precipitantes se destacaron los siguientes: progresión de falla cardiaca (27\%), falla cardiaca de novo (26\%), falta de adherencia (22,6\%), fibrilación atrial $(6 \%)$ e infección $(5,3 \%)$. Las etiologías más frecuentes de la falla cardiaca fueron, en primer lugar, la hipertensiva (31,6\%), seguida de la causa isquémica $(24,3 \%)$ y por último la valvular $(14,5 \%)$. El promedio de puntaje en la escala clínica de Boston fue de 9,5. La mediana de la fracción de expulsión fue del 25\% (tabla 2).

\section{Presentación clínica}

El promedio de la presión arterial sistólica fue de $126 \pm 29,6 \mathrm{~mm} \mathrm{Hg}$ y la proporción de la presión de pulso de 39\%; la clase funcional de acuerdo con la Asociación del Corazón de Nueva York (NYHA, su sigla en inglés por New York Heart Association) fue en su mayoría clase III (44,6\%) y clase IV (45\%). Los hallazgos más comunes hechos durante el examen físico fueron el edema de miembros inferiores
Tabla 3 Presentación clínica

\begin{tabular}{|c|c|}
\hline Variable clínica & $\mathrm{n}=247$ \\
\hline Frecuencia cardiaca (latidos/minuto, $X \pm D E$ ) & $91,6 \pm 22$ \\
\hline Presión arterial sistólica ( $\mathrm{mm} \mathrm{Hg}, X \pm D E$ ) & $126 \pm 29,6$ \\
\hline Presión arterial diastólica ( $\mathrm{mm} \mathrm{Hg}, X \pm D E)$ & $77,4 \pm 18,2$ \\
\hline Presión de pulso ( $m m \mathrm{Hg}, X \pm D E$ ) & $49,0 \pm 19$ \\
\hline Proporción de presión de pulso $(\%, X \pm D E)$ & $39,5 \pm 11,5$ \\
\hline \multicolumn{2}{|l|}{ Clase funcional de la NYHA (\%): } \\
\hline III & 44,6 \\
\hline IV & 45 \\
\hline Edema de miembros inferiores (\%) & 68,8 \\
\hline Ingurgitación yugular a $45^{\circ}(\%)$ & 63,8 \\
\hline Crépitos pulmonares (\%) & 52,2 \\
\hline Hepatomegalia (\%) & 36 \\
\hline Reflujo hepatoyugular presente (\%) & 20,6 \\
\hline Frialdad en extremidades (\%) & 11,7 \\
\hline Galope por S3 (\%) & 9,3 \\
\hline Alteración del sensorio (\%) & 5,7 \\
\hline
\end{tabular}

Tabla 4 Paraclínicos

\begin{tabular}{|c|c|}
\hline Laboratorio & $n=247$ \\
\hline Sodio sérico inicial (mmol/L, $X \pm D E$ ) & $138 \pm 4,25$ \\
\hline Potasio sérico inicial (mmol/L, $\mathrm{X} \pm \mathrm{DE}$ ) & $4,40 \pm 0,71$ \\
\hline BUN (mg/dl, Me, Q1-Q3) & $23,5(17-35)$ \\
\hline Creatinina (mg/dl, Me, Q1-Q3) & $1,17(0,9-1,6)$ \\
\hline Hemoglobina (g/dl, $\mathrm{X} \pm \mathrm{DE})$ & $12,9 \pm 2,45$ \\
\hline Hematocrito $(\%, X \pm D E)$ & $39,8 \pm 7,54$ \\
\hline $\begin{array}{l}\text { Tasa de filtración glomerular por MDRD } \\
\left(\mathrm{ml} / \mathrm{min} \times 1,73 \mathrm{~m}^{2} \mathrm{SC}, \mathrm{X} \pm \mathrm{DE}\right)\end{array}$ & $60,6 \pm 28,8$ \\
\hline
\end{tabular}

$(68,8 \%)$, la ingurgitación yugular a $45^{\circ}(63,8 \%)$, los crépitos pulmonares (52\%) y la hepatomegalia (36\%) (tabla 3$)$.

\section{Paraclínicos}

Se destacan el sodio sérico con promedio de $138 \mathrm{mmol} / \mathrm{L}$, el potasio sérico con 4,4 mmol/L, el BUN con $23,5 \mathrm{mg} / \mathrm{dl}$ y la creatinina con $1,17 \mathrm{mg} / \mathrm{dl}$. La tasa de filtración glomerular promedio estimada por fórmula MDRD (del inglés Modification of Diet in Renal Disease) fue de $60,6 \mathrm{ml} / \mathrm{min}$ (tabla 4).

\section{Manejo intrahospitalario y al egreso}

Se requirió uso de soporte inotrópico y vasopresor en el $15,8 \%$ y $12 \%$, respectivamente (tabla 5 ). El soporte circulatorio con balón de contrapulsación intraaórtica u oxigenación por membrana extracorpórea (ECMO) fue necesario en 1,6\% y $0,4 \%$, respectivamente. Los medicamentos más usados durante la hospitalización y al momento del egreso hospitalario fueron, en orden de frecuencia, diurético de asa tipo furosemida $(88,7 \%)$, inhibidores de la enzima convertidora de angiotensina (IECA)/antagonistas del receptor de angiotensina tipo II (ARA II) (85\%), betabloqueadores (82\%), antialdosterónicos $(58,7 \%)$ y digoxina $(18,6 \%)$. 
Tabla 5 Manejo intrahospitalario y al egreso

\begin{tabular}{ll}
\hline Medicamento & $\mathrm{n}=247$ \\
\hline IECA (\%) & 64 \\
ARA II (\%) & 21,1 \\
Diurético de asa (\%) & 88,7 \\
Betabloqueador (\%) & 82,2 \\
Antialdosterónico (\%) & 58,7 \\
Digoxina (\%) & 18,6 \\
Inotrópicos (\%) & 15,8 \\
Vasopresores (\%) & 12,1 \\
Soporte circulatorio (\%): & \\
Balón de contrapulsación intraaórtica & 1,6 \\
Oxigenación por membrana extracorpórea & 0,4 \\
\hline
\end{tabular}

Tabla 6 Resultados clínicos durante la hospitalización y en el seguimiento a treinta días y seis meses

\begin{tabular}{ll}
\hline Desenlace & $\mathrm{n}=247$ \\
\hline Mortalidad intrahospitalaria (\%) & 9,3 \\
Mortalidad acumulada a 30 días (\%) & 10,9 \\
Mortalidad acumulada a 6 meses (\%) & 14,1 \\
Complicaciones intrahospitalarias (\%): & \\
Choque & 14,1 \\
Lesión renal aguda & 10,4 \\
Necesidad de ventilación mecánica invasiva & 3,2 \\
Accidente cerebrovascular & 1,2 \\
Estancia hospitalaria (días, Me, Q1-Q3) & $9(5-16)$ \\
Ingreso a unidad de cuidados intensivos (\%) & 19,8 \\
\hline
\end{tabular}

\section{Resultados clínicos durante la hospitalización y en el seguimiento a treinta días y seis meses}

La mediana de la estancia hospitalaria fue de nueve días. El ingreso a unidad de cuidados intensivos ( $\mathrm{UCl}$ ) fue de $19,8 \%$. Las complicaciones más frecuentes fueron choque (14\%), lesión renal aguda $(3,2 \%)$ y accidente cerebrovascular (1,2\%). La mortalidad intrahospitalaria fue de $9,3 \%$, mientras que la acumulada a treinta días fue de $10,9 \%$ y a seis meses de $14,1 \%$ (tabla 6$)$.
Tabla 7 Regresión logística de mortalidad intrahospitalaria vs. BUN y PAS

\begin{tabular}{llll}
\hline Variable & OR $(95 \%$ IC $)$ & Wald X2 & Valor de $\mathrm{p}$ \\
\hline BUN $>37 \mathrm{mg} / \mathrm{dl}$ & $10,8(4,10-28,8)$ & 23,0 & 0,000 \\
PAS $\leq 125 \mathrm{~mm} \mathrm{Hg}$ & $3,42(1,15-10,0)$ & 4,94 & 0,026 \\
\hline
\end{tabular}

\section{Análisis de mortalidad ajustada}

Según criterios clínicos se identificaron cinco potenciales variables candidatas como predictores de mortalidad intrahospitalaria. Al realizar el análisis univariado se encontraron diferencias significativas entre ambos grupos por mortalidad hospitalaria para el BUN al ingreso (valor p 0,000) y presión arterial sistólica (valor p 0,000), con relación lineal entre la probabilidad de muerte intrahospitalaria y los niveles de estas dos variables (fig. 1), mientras que para las variables sodio sérico al ingreso, etiología isquémica de la falla cardiaca y la presencia de bloqueo completo de la rama izquierda del haz de His no se encontraron diferencias significativas para ingresar estas variables al modelo.

Entre las variables ingresadas al modelo, la regresión logística multivariada identificó los dos predictores de riesgo estadísticamente significativos para explicar la mortalidad intrahospitalaria, los cuales fueron BUN > $37 \mathrm{mg} / \mathrm{dl}$ y presión arterial sistólica $\leq 125 \mathrm{~mm} \mathrm{Hg}$ (tabla 7). La prueba de bondad de ajuste de Hosmer-Lemeshow para este modelo arrojó un valor p 0,246 siendo indicador de buen ajuste mientras que el área bajo la curva ROC fue de 0,769 , que para modelos predictivos muestra buena capacidad para clasificar correctamente a los pacientes.

El modelo CART determina como puntos de corte niveles de BUN de $32,5 \mathrm{mg} / \mathrm{dl}$, presión arterial sistólica $97 \mathrm{~mm} \mathrm{Hg}$ y niveles de creatinina de $1,75 \mathrm{mg} / \mathrm{dl}$, con lo cual estratifica el riesgo en bajo, intermedio o alto; un nivel de BUN al ingreso $<32,5 \mathrm{mg} / \mathrm{dl}$ clasifica al paciente como de bajo riesgo (mortalidad $0,53 \%$ ), un nivel de BUN $\geq 32,5 \mathrm{mg} / \mathrm{dl}$ con presión arterial sistólica $\geq 97 \mathrm{~mm} \mathrm{Hg}$ o $\mathrm{BUN} \geq 32,5 \mathrm{mg} / \mathrm{dl}$ con presión arterial sistólica $<97 \mathrm{~mm} \mathrm{Hg}$ y creatinina $<1,75 \mathrm{mg} / \mathrm{dl}$ como riesgo intermedio (mortalidad 14,3-21,6\%) y BUN $\geq 32,5 \mathrm{mg} / \mathrm{dl}$ con presión arterial sistólica $<97 \mathrm{~mm} \mathrm{Hg}$ y creatinina $\geq 1,75 \mathrm{mg} / \mathrm{dl}$ como riesgo alto (mortalidad $76,9 \%$ ) (fig. 2).

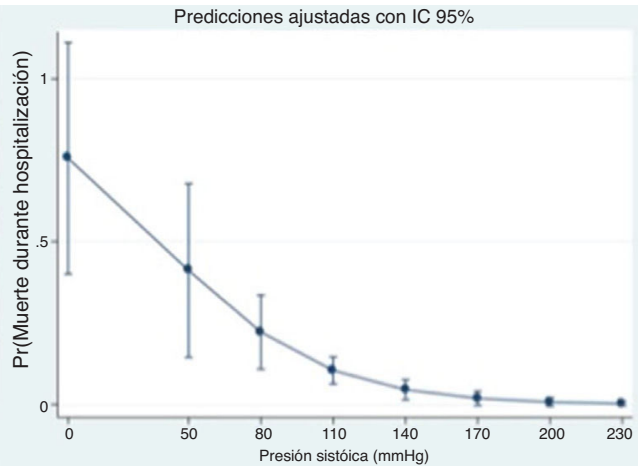

Figura 1 Relación entre el BUN al ingreso y la presión arterial sistólica, con la probabilidad de muerte. 


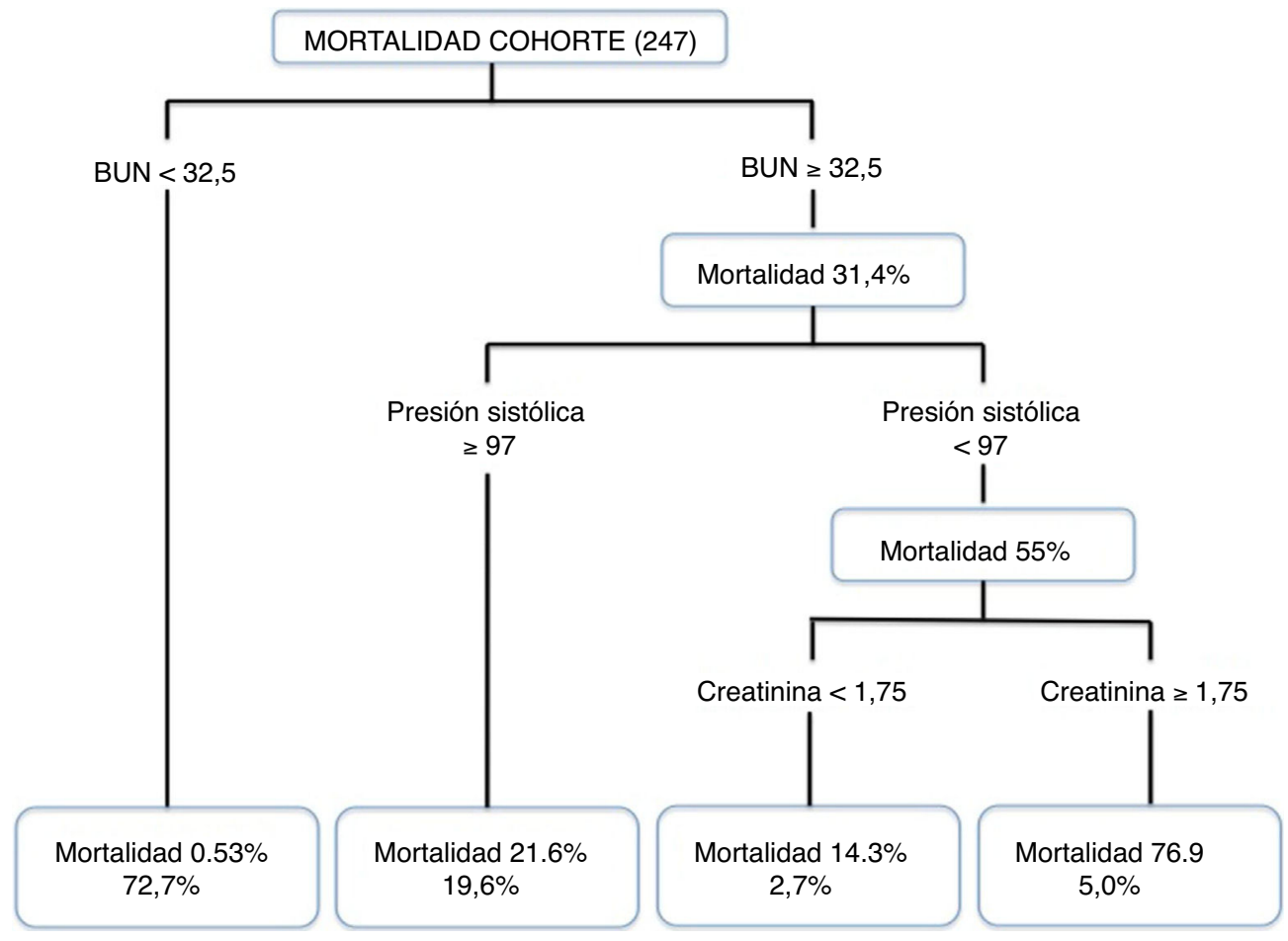

Figura 2 Modelo CART para estratificación de riesgo de muerte intrahospitalaria.

\section{Discusión}

El análisis de esta cohorte provee información sobre las características de una población de pacientes admitidos a hospitalización por falla cardiaca aguda y determina algunas variables no invasivas al momento del ingreso, que en conjunto con datos de laboratorio, de uso rutinario, ayudan en la predicción temprana del riesgo de muerte en estos pacientes. Tanto la mortalidad intrahospitalaria $(9,3 \%)$ como la acumulada a 30 días $(10,9 \%)$ y 6 meses $(14,1 \%)$ concuerda con datos hallados en otros estudios ${ }^{3,6,7}$; sin embargo, la estratificación permite establecer rangos de mortalidad bastante amplios $(0,5-77 \%)$, teniendo en cuenta que al año puede ser mayor en pacientes con falla cardiaca aguda ${ }^{23}$.

Las variables asociadas al riesgo de muerte intrahospitalaria encontradas en el presente estudio (BUN y presión arterial sistólica) coinciden con descripciones en otros estudios $^{2,3,12,20,24}$. La presión arterial sistólica al ingreso es un factor de riesgo independiente asociado a morbilidad y mortalidad en falla cardiaca tanto con fracción de expulsión reducida como preservada ${ }^{24}$ y puede ayudar en la estratificación temprana y a diferenciar entre grupos de pacientes que tienen características clínicas e incluso de tratamiento distintos.

La importancia de la presión arterial sistólica en la estratificación temprana al ingreso se refleja en el algoritmo recientemente propuesto por las "Guías de falla cardiaca de la Sociedad Europea de Cardiología",25. Lee et al., con base en la población del estudio Enhaced Feedback For Efective Cardiac Treatment (EFFECT) idearon un modelo de regresión logística multivariado para predicción de mortalidad a treinta días y un año. La disminución de la presión arterial sistólica (por cada $10 \mathrm{~mm} \mathrm{Hg}$ ) y la elevación del BUN (por cada aumento de $10 \mathrm{mg} / \mathrm{dl}$ ) fueron predictores independientes y significativos, tanto para mortalidad intrahospitalaria como a un año. El área bajo la curva ROC del modelo fue de 0,8 para la mortalidad a 30 días y 0,77 para la mortalidad a 1 año ${ }^{7}$. En nuestro estudio tener una presión arterial sistólica $\leq 125 \mathrm{~mm} \mathrm{Hg}$ al momento del ingreso aumentó en 3,4 veces el riesgo de muerte intrahospitalaria. Fonarow et al. ${ }^{12}$, en el estudio ADHERE identificaron el BUN, la creatinina y la presión arterial sistólica como los mejores predictores de mortalidad intrahospitalaria usando el análisis por CART. Dicho modelo es atractivo puesto que clasifica los pacientes en tres categorías de riesgo (bajo, intermedio y alto) con tan solo tres variables. De forma similar, en una revisión retrospectiva de 1.004 pacientes hospitalizados por falla cardiaca, el empeoramiento de la función renal se asoció con un incremento de 7,5 veces (IC 95\%, 2,9-19,3 veces) en el riesgo ajustado de mortalidad intrahospitalaria ${ }^{26}$. Se ha evidenciado que la disfunción renal causa mayor congestión y activación neurohormonal, factores asociados con eventos adversos en pacientes con falla cardiaca ${ }^{27}$. En nuestro estudio, tener un nivel de BUN> $37 \mathrm{mg} / \mathrm{dl}$ al momento del ingresó aumentó diez veces el riesgo de muerte.

Además de los parámetros mencionados, otras variables han sido correlacionadas con desenlaces en pacientes hospitalizados con falla cardiaca, las cuales incluyen: edad ${ }^{10}$, sexo ${ }^{27}$, etiología de la falla cardiaca ${ }^{27}$, hospitalizaciones previas por falla cardiaca ${ }^{28}$, condiciones comórbidas (enfermedad cerebrovascular ${ }^{7}$, diabetes ${ }^{10}$, demencia, enfermedad pulmonar obstructiva crónica (EPOC), cirrosis hepática y cáncer $\left.^{7}\right)$, frecuencia respiratoria ${ }^{7}$, anemia ${ }^{10}$, sodio sérico ${ }^{7}$, niveles de péptido natriurético tipo $B(B N P)^{29,30}$, fracción de expulsión $^{31}$ y terapia para falla cardiaca ${ }^{32}$. 
La capacidad para clasificar a los pacientes en categorías de alto riesgo puede guiar la inclusión en programas de manejo de enfermedades o seguimiento intensivo temprano, intervenciones que potencialmente son más costosas, pero pueden ser costoefectivas para aquellos pacientes ubicados en el riesgo más alto ${ }^{29}$. Con los datos del módulo de la Asociación Americana del Corazón (AHA, su sigla en inglés por American Heart Association y GWTG-HF (Get With The Guidelines-Heart Failure) ${ }^{29}$, creado como una iniciativa voluntaria para recolección de datos de pacientes que son hospitalizados por falla cardiaca en más de doscientos hospitales afiliados en Estados Unidos, Peterson et al. desarrollaron un modelo pronóstico para predicción del riesgo de muerte intrahospitalaria por falla cardiaca. Tanto en la cohorte de derivación como en la cohorte de validación, la capacidad discriminativa del modelo fue intermedia (estadístico $C=0,75)$. De acuerdo con el puntaje de riesgo (0-100), la mortalidad intrahospitalaria predicha varió desde $0,4 \%$ hasta $9,7 \%$. El puntaje de riesgo GWTG-HF está disponible como calculadora en línea (http: / / www. americanheart.org/presenter.jhtml?identifier 3027533) ${ }^{27}$.

Kociol et al. ${ }^{30}$, describieron, con base en el registro OPTIMIZE-HF (del inglés Organized Program to Initiate Lifesaving Treatment in Hospitalized Patients With Heart Failure), como variables predictoras de mortalidad a un año, los niveles de BNP al egreso hospitalario, la edad (por cada diez años), la presión arterial al ingreso y el antecedente de EPOC. El BNP al egreso fue la variable con mayor poder predictivo de muerte y el compuesto de muerte $u$ hospitalización a un año. El aumento de peso, los niveles de hemoglobina y sodio sérico y el antecedente personal de hiperlipidemia se consideraron como factores protectores ${ }^{29}$. La adición del BNP al egreso al modelo de desenlaces a largo plazo mejora la capacidad de estratificar de manera adecuada a los pacientes en categorías de bajo, medio y alto riesgo, definidas por variables clínicas y paraclínicas estándar. OĆonnor et al., idearon un modelo pronóstico en pacientes con falla cardiaca avanzada del estudio ESCAPE (del inglés Evaluation Study of Congestive Heart Failure and Pulmonary Artery Catheterization Effectiveness) ${ }^{3}$. La mortalidad a seis meses fue de $18,7 \%$. El estadístico $C$ del modelo fue de 0,76 , cifra que sugiere una capacidad razonablemente buena para discriminar entre pacientes fallecidos y supervivientes a seis meses. El área bajo la curva ROC de nuestro modelo fue de 0,769 , que para modelos predictivos muestra buena capacidad para clasificar correctamente a los pacientes que sobreviven de los fallecidos. En nuestro estudio, otras variables clásicamente asociadas con mortalidad intrahospitalaria no alcanzaron la significancia estadística, tales como sodio sérico al ingreso, etiología de la falla cardiaca y bloqueo de rama izquierda. La utilidad de biomarcadores ampliamente conocidos por su valor pronóstico, como el BNP y el NT-proBNP29,30,33,34, no pudo ser evaluada en nuestro estudio puesto que estos no se realizan de forma rutinaria.

Mediante la utilización de tres variables (BUN, presión sistólica y creatinina) al ingreso del paciente, el modelo CART permite clasificar la probabilidad del riesgo de muerte intrahospitalaria, en una forma simple de máximo tres pasos, mucho más fácil de interpretar. Los puntos de corte encontrados pueden ser sensibles a la muestra estudiada y deben validarse en una población de mayor tamaño. La determinación de un nivel de BUN $<32 \mathrm{mg} / \mathrm{dl}$ estratifica, de entrada, un grupo de bajo riesgo, mientras que un $B U N \geq 32 \mathrm{mg} / \mathrm{dl}$, presión sistólica $<97 \mathrm{~mm} \mathrm{Hg}$ y creatinina $\geq 1,75 \mathrm{mg} / \mathrm{dl}$ uno de alto riesgo. Aunque está claro que el modelo solo clasifica el riesgo de muerte intrahospitalaria, puede convertirse en una herramienta útil para que el clínico determine la necesidad de monitorización invasiva o no invasiva, seguimiento clínico y paraclínico y estrategias terapéuticas.

\section{Limitaciones}

Este estudio de cohorte fue hecho en un solo centro hospitalario, de modo que puede haber sesgo de referencia. El tamaño de la muestra es pequeño comparado con los grandes registros de falla cardiaca; sin embargo, es suficiente para el número de variables predictoras analizadas en ambos modelos. El análisis retrospectivo de los datos puede incluir sesgos de información, aunque la vinculación de la mayoría de ellos al programa de clínica de falla cardiaca, la elaboración de una historia estructurada y el seguimiento estricto hicieron que las pérdidas fueran mínimas y la calidad del dato alta.

Los biomarcadores cardiacos conocidos por su valor pronóstico no fueron evaluados en este estudio de cohorte debido a la baja frecuencia con la que se realiza al ingreso de los pacientes con falla cardiaca, dado que están indicados en pacientes con dudas diagnósticas según las guías de práctica clínica y no se emplean de forma rutinaria ${ }^{35}$.

\section{Conclusión}

El análisis mediante el modelo de árbol de regresión y clasificación (CART) permite catalogar en forma temprana la probabilidad de muerte por un árbol de riesgo que incluye el $\mathrm{BUN} \geq 32,5 \mathrm{mg} / \mathrm{dl}$, presión sistólica $<97 \mathrm{~mm} \mathrm{Hg}$ y los niveles de creatinina $\geq 1,75 \mathrm{mg} / \mathrm{dl}$. La identificación de los pacientes con mayor riesgo de muerte intrahospitalaria puede orientar la intervención y monitorización más agresiva en este grupo de pacientes.

\section{Financiación}

La investigación fue financiada con recursos propios del Grupo para el Estudio de las Enfermedades Cardiovasculares, de la sección de Cardiología de la Universidad de Antioquia.

\section{Conflictos de interés y aspectos éticos}

No hay conflictos de interés por declarar.

Los aspectos éticos se ajustan a los principios éticos fundamentales y a las directrices de la Declaración de Helsinki y similares, emanados de la Asociación Médica Mundial, y sigue las pautas del Ministerio de la Protección Social de la República de Colombia según la resolución 8430 de 1993 por la cual se dictan las normas científicas, técnicas y administrativas para la investigación en salud. 


\section{Bibliografía}

1. Hsiao R, Greenberg B. Contemporary treatment of acute heart failure. Prog Cardiovasc Dis. 2016;58:367-78, http://dx.doi.org/10.1016/j.pcad.2015.12.005.

2. Abraham WT, Fonarow GC, Albert NM, Stough WG, Gheorghiade $\mathrm{M}$, MGreenberg $\mathrm{BH}$, et al. Predictors of In-Hospital Mortality in Patients Hospitalized for Heart Failure: Insights From the Organized Program to Initiate Lifesaving Treatment in Hospitalized Patients With Heart Failure (OPTIMIZE-HF). J Am Coll Cardiol. 2008;52:347-56, http://dx.doi.org/10.1016/j.jacc.2008.04.028.

3. O'Connor CM, Hasselblad V, Mehta RH, Tasissa G, Califf RM, Fiuzat $M$, et al. Triage after hospitalization with advanced heart failure: The ESCAPE (Evaluation Study of Congestive Heart Failure and Pulmonary Artery Catheterization Effectiveness) risk model and discharge score. J Am Coll Cardiol. 2010;55:872-8, http://dx.doi.org/10.1016/j.jacc.2009.08.083.

4. Sénior JM, Saldarriaga CI, Rendón JA. Descripción clínicoepidemiológica de los pacientes con falla cardiaca aguda que consultan al servicio de urgencias. Acta Med Colomb. 2011;36:125-9.

5. Chaves W, Diaztagle J, Sporckel JJ, Hernández J, Benavidez JM, Henao DC, et al. Factores asociados a mortalidad en pacientes con falla cardiaca descompensada. Acta Med Colomb. 2014;39:314-20.

6. Tomcikova D, Felsoci M, Spinar J, Miklik R, Mikusova T, Vitovec J, et al. Risk of in-hospital mortality identified according to the typology of patients with acute heart failure: Classification tree analysis on data from the Acute Heart Failure Database-Main registry. J Crit Care. 2013;28:250-8, http://dx.doi.org/10.1016/j.jcrc.2012.09.014.

7. Lee DS, Austin PC, Rouleau JL, Liu PP, Naimark D, Tu JV. Predicting mortality among patients hospitalized for heart failure: derivation and validation of a clinical model. JAMA. 2003;290:2581-7, http://dx.doi.org/10.1001/jama.290.19.2581.

8. Harrel FE, Lee KL, Mark DB. Multivariable prognostic models: issues in developing models, evaluating assumptions and adequacy and measuring and reducing errors. Stat Med. 1996;15:361-87.

9. Steyerberg EW, Eijkemans MJ, Harrell FE, Habbema DF. Prognostic modelling with logistic regression analysis: a comparison of selection and estimation methods in small data sets. Stat Med. 2000;19:1059-79.

10. Spinar J, Jarkovsky J, Spinarova L, Mebazaa A, Gayat E, Vitovec J, et al. AHEAD score - Long-term risk classification in acute heart failure. Int J Cardiol. 2016;202:21-6, http://dx.doi.org/10.1016/j.ijcard.2015.08.187.

11. Vazquez R, Bayes A, Cygankiewicz I, Pascual D, Grigorian L, Pavon R, et al. The MUSIC Risk score: a simple method for predicting mortality in ambulatory patients with chronic heart failure. Eur Heart J. 2009;30:1088-96, http://dx.doi.org/10.1093/eurheartj/ehp032.

12. Fonarow GC, Adams KF, Yancy CW, Boscardin WJ. Risk stratification for in-hospital mortality in acutely decompensated heart failure: classification and regression tree analysis. JAMA. 2005;293:572-80, http://dx.doi.org/10.1001/jama.293.5.572.

13. Burgueño MJ, García JL, González JM. Las curvas ROC en la evaluación de las pruebas diagnósticas. Med Clin. 1995;104:661-70.

14. Steyerberg E, Vergouwe I. Towards better clinical prediction models: seven steps fopr development and an $A B C D$ for validation. Eur Heart J. 2014;35:1925-31, http://dx.doi.org/10.1093/eurheartj/ehu207.

15. Carlson KJ, Lee DC, Goroll AH, Leahy M, Johnson RA. An Analysis of physicianŕeasons for preescribing long-term digitalis therapy in outpatients. J Chron Dis. 1985;38:733-9.
16. Marantz PR, Tobin JN, Wassertheil S, Steingart RM, Wexler JP, Budner $N$. The relationship between left ventricular systolic function and congestive heart failure diagnosed by clinical criteria. Circulation. 1988;77:607-12, http://dx.doi.org/10.1161/CIR.0b013e31829e8776.

17. Yancy C, Jessup M, Bozkurt B, Butler J, Casey D, Drazner $M$, et al. 2013 ACCF/AHA Guideline for the Management of Heart Failure: A Report of the American College of Cardiology Foundation/American Heart Association Task Force on Practice Guidelines. Circulation. 2013;128:e240-327, http: //dx.doi.org/10.1161/CIR.0b013e3.

18. Nohria A, Tsang SW, Fang JC, Lewis EF, Jarcho JA, Stevenson LW, et al. Clinical assessment identifies hemodynamic profiles that predict outcomes in patients admitted with heart failure. J Am Coll Cardiol. 2003;41:1797-804, http://dx.doi.org/10.1016/S0735-1097(03)00309-7.

19. Ministerio de Salud y Protección Social de Colombia. [Internet]. Resolución 8430 de 1993;1-19. [Acceso 29 Abr 2018]. Disponible en: https://www.minsalud.gov.co/sites/ rid/Lists/BibliotecaDigital/RIDE/DE/DIJ/RESOLUCION-8430DE-1993.PDF.

20. Yancy CW, Lopatin M, Stevenson LW, De Marco T, Fonarow GC. Clinical presentation, management and in-hospital outcomes of patients admitted with acute descompensated heart failure with preserved systolic function: a report from the Acute Descompensated Heart Failure National (ADHERE) Database. J Am Coll Cardiol. 2006;47:76-84, http://dx.doi.org/10.1016/j.jacc.2005.09.022.

21. Zelterman D. Discrimination and classification. En: Zelterman D Applied Multivariate Statistics with R. Switzerland: Springer; 2015. p. 257-86.

22. Lewis RJ. An introduction to classification and regression tree (CART) analysis [Internet]. Department of Emergency Medicine, Harbor-UCLA Medical Center, Torrance, California. [Acceso 29 Abr 2018. Disponible en: http: //www.saem.org/download/lewis1.pdf.

23. Crespo-Leiro MG, Anker SD, Maggioni AP, Coats AJ, Filippatos G, Ruschitzka F, et al. European Society of Cardiology Heart Failure Long-Term Registry (ESC-HF-LT): 1-year follow-up outcomes and differences across regions. Eur J Heart Fail. 2016;18:613-25, http: //dx.doi.org/10.1002/ejhf.566.

24. Gheorghiade $M$, Abraham WT, Albert NM, Greenberg BH, OĆonnor CM, She L, et al. Systolic blood pressure at admission, clinical characteristics, and outcomes in patients hospitalized with acute heart failure. JAMA. 2006;296:2217-26, http://dx.doi.org/10.1001/jama.296.18.2217.

25. Ponikowski P, Voors AA, Anker SD, Bueno H, Cleland JG, Coats AJ, et al., Guía ESC. 2016 sobre el diagnóstico y tratamiento de la insuficiencia cardiaca aguda y crónica. Grupo de Trabajo de la Sociedad Europea de Cardiología (ESC) de diagnóstico y tratamiento de la insuficiencia cardiaca aguda y crónica. Rev Esp Cardiol. 2016;69:1167, http://dx.doi.org/10.1016/j.recesp.2016.09.056, e1-e85.

26. Forman DE, Butler J, Wang Y, Abraham WT, O'Connor CM, Gottielb SS, et al. Incidence, predictors at admission, and impact of worsening renal function among patients hospitalized with heart failure. J Am Coll Cardiol. 2004;43:61-7, http://dx.doi.org/10.1016/j.jacc.2003.07.031.

27. Bongartz LG, Cramer MJ, Braam B. The cardiorenal connection. Hypertension. 2004;43:e14. DOI: 10.1161/01.HYP. 0000118521.06245.b8.

28. Felker GM, Gattis WA, Leimberger JD, Adams KF, Cuffe MS, Gheorghiade $M$, et al. Usefulness of anemia as a predictor of death and rehospitalization in patients with decompensated heart failure. Am J Cardiol. 2003;92:625-8, http://dx.doi.org/10.1016/S0002-9149(03)00740-9.

29. Peterson PN, Rumsfeld JS, Liang L, Albert NM, Hernandez AF, Peterson ED. A validated risk score for in-hospital mortality in 
patients with heart failure from the American Heart Association get with the guidelines program. Circ Cardiovasc Qual Outcomes. 2010;3:25-32. DOI: 10.1161/CIRCOUTCOMES.109.854877.

30. Kociol RD, Horton JR, Fonarow GC, Reyes EM, Shaw LK, O'Connor $\mathrm{CM}$, et al. Admission, Discharge, or Change in B-Type Natriuretic Peptide and Long-Term Outcomes: Data From Organized Program to Initiate Lifesaving Treatment in Hospitalized Patients With Heart Failure (OPTIMIZE-HF) Linked to Medicare Claims. Circ Heart Fail. 2011;4:628-36. DOI: 10.1161/CIRCHEARTFAILURE.111.962290.

31. Kerzner R, Gage BF, Freedland KE, Rich MW. Predictors of mortality in younger and older patients with heart failure and preserved or reduced left ventricular ejection fraction. Am Heart J. 2003;146:286-90, http://dx.doi.org/10.1016/S0002-8703(03)00151-0.

32. Clinical Quality Improvement Network Investigators. Mortality risk and patterns of practice in 4606 acute care patients with congestive heart failure: the relative importance of age, sex, and medical therapy. Arch Int Med. 1996;156:1669-73. DOI: 10.1001/archinte.1996.00440140095009.
33. Herrero P, Prieto B, García M, Jacob J, Martín FJ, Pascual $D$, et al. Predictive capacity of a multimarker strategy to determine short-term mortality in patients attending a hospital emergency Department for acute heart failure. BIO-EAHFE study. Clinical Chimica Acta. 2017;466:22-30, http://dx.doi.org/10.1016/j.cca.2017.01.003.

34. Lassus J, Gayat E, Mueller C, Peacok WF, Spinar J, Harjola VP, et al. Incremental value of biomarkers to clinical variables for mortality prediction in acutely decompensated heart failure: The Multinational Observational Cohort on Acute Heart Failure (MOCA) study. Int J Cardiol. 2013;168:2186-94, http://dx.doi.org/10.1016/j.ijcard.2013.01.228.

35. Colombia. Ministerio de Salud y Protección Social [Internet]. Guía de Práctica Clínica ?(GPC) para la prevención, diagnóstico, tratamiento y rehabilitación de la falla cardíaca en población Mayr de 18 años clasificación B, C y D. Guía completa No 53 [GPC en Internet]. Edición 1a. Bogotá DC: El Ministerio; 2016 [Acceso 29 Abr de 2018. Disponible en: http://gpc.minsalud.gov.co/gpc_sites/Repositorio/Conv 637/GPC_falla_cardiaca/gpc_falla_cardiaca_completa.aspx. 